\title{
NUMBER COUNTS AND SPECTRAL DISTRIBUTION OF RADIO SOURCES AT CENTIMETER WAVELENGTHS
}

\author{
I. I. K. PAULINY-TOTH*, K. I. KELLERMANN and M. M. DAVIS \\ National Radio Astronomy Observatory, ${ }^{* *}$ Green Bank, West Va., U.S.A.
}

\begin{abstract}
The results of a survey of selected regions of sky made at a frequency of $5 \mathrm{GHz}$, and of measurements of the spectral index distributions for sources selected from low frequency surveys are presented. The source counts and spectral distribution at $5 \mathrm{GHz}$ are in excellent agreement with those expected from surveys made at lower frequencies. There is no significant dependence of the spectral distribution on flux density in any of the surveys we have investigated, implying either a low redshift for the sources, or a systematic change in their properties with redshift.
\end{abstract}

In 1968 , a survey of selected regions of sky at a frequency of $5 \mathrm{GHz}(\lambda=6 \mathrm{~cm})$ was begun at the National Radio Astronomy Observatory. A preliminary report (Kellermann et al., 1968, hereinafter referred to as Paper I) showed that the source counts and spectral distribution of this and other surveys at centimeter and decimeter wavelengths were consistent with the data from surveys at meter wavelengths, in contradiction to the conclusions of Shimmins et al. (1968).

The present paper reports on an extension of the $5 \mathrm{GHz}$ survey and of the spectral measurements. It is hoped that this survey will ultimately cover most of the northern sky to a moderate flux level and that measurements of accurate positions, optical identifications and observations of source structure will lead to a picture of the sky at centimeter wavelengths similar to that now available at meter wavelengths.

A particular aim is to look for a distance indicator in the radio data alone. This may be provided by the dependence of the radio ' $K$-correction' on the spectral index, $\alpha$ of the source. This correction is given by

$$
S^{\prime}=S^{\prime}(1+z)^{1+\alpha}
$$

where $S^{\prime}$ is the flux density in the rest-frame of the source, $S$ is the observed flux density and $\alpha$ is defined by $S \propto$ (frequency) ${ }^{\alpha}$. This correction is small for sources with $\alpha \sim-1$, that is, for almost all sources detected in surveys at meter wavelengths, but can be large for sources with $\alpha \sim 0$, which form a large fraction of sources found by surveys made at centimeter wavelengths. The effect of this term is that the proportion of sources with flat spectra in any survey is expected to increase with increasing redshift. If, for example, for sources with $\alpha \sim-1$, a number-flux relation $N(S)=K S^{x}$ is observed, then the proportion of sources with $\alpha \sim 0$, for a typical redshift $z=2$ and for $x=-1.5$, is expected to increase by a factor of about 5 over its value at low redshifts. A further effect is that the redshifts of sources with $\alpha \sim 0$ should become progressively higher, compared to those of sources with $\alpha \sim-1$ as the flux density decreases.

In practice, the magnitude of the effect is difficult to assess for several reasons. First, because of the wide range in the absolute radio luminosities, there is a large

* Present address: Max-Planck-Institut für Radioastronomie, Bonn, Germany.

** Operated by Associated Universities, Inc., under contract with the National Science Foundation. 
dispersion in the redshift at a particular flux level. Second, the luminosity function or the spatial distribution of sources with flat spectra may not be the same as that of sources with steep spectra. Third, the luminosity function may vary with the redshift in a different way for the two types of sources. In particular, if the luminosity function is independent of $\alpha$ at one frequency, it cannot be so at any other frequency.

\section{The $5 \mathrm{GHz}$ Survey}

The survey was made with the $140-\mathrm{ft}$ telescope of the National Radio Astronomy Observatory in 1969-70. The receiving equipment and observing techniques were similar to those reported in Paper I. Three, rather than two, independent surveys were

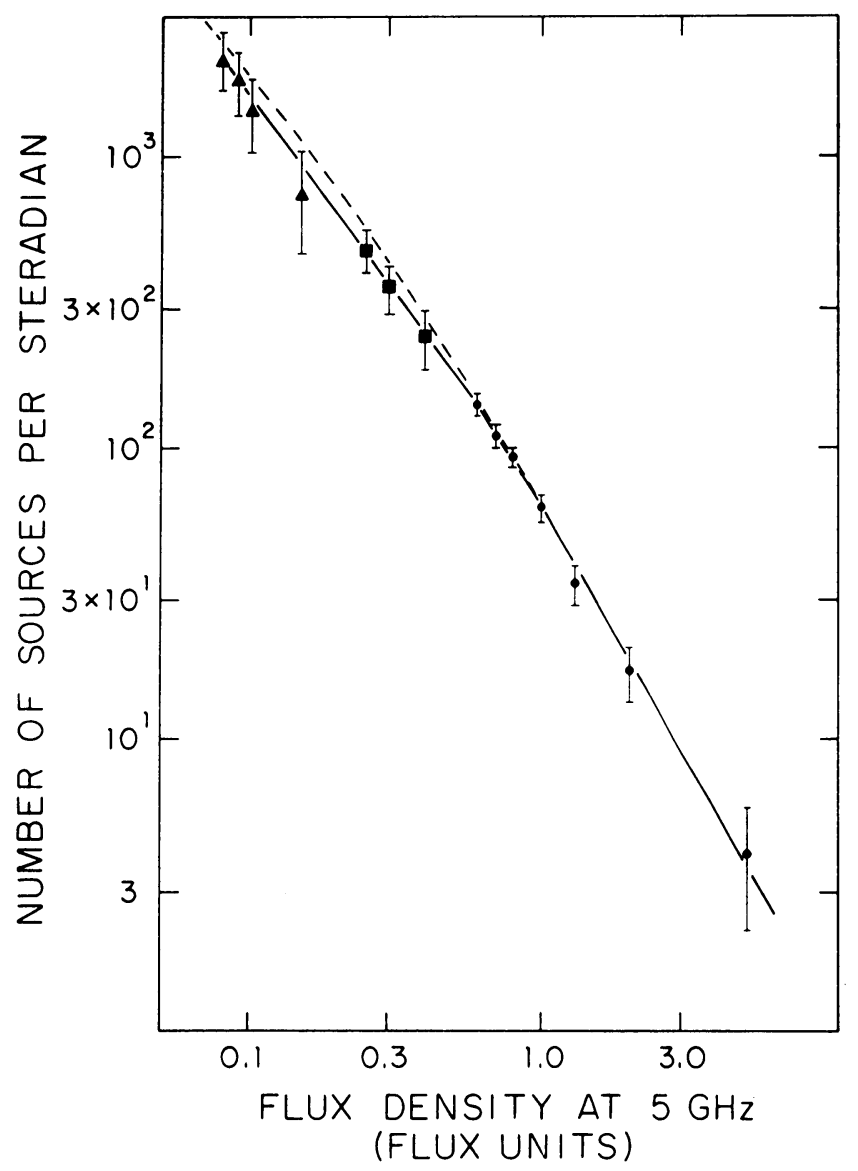

Fig. 1. Source counts for the $5 \mathrm{GHz}$ survey; points represent the number of sources with flux density equal to or greater than the given value. Circles, squares and triangles refer to the 'fast', 'medium' and 'deep' surveys respectively; the errors shown are not independent within a survey but are independent from one survey to another. Solid lines represent best fits for $S \geqslant 1$ f.u., $S \geqslant 0.6$ f.u., and the slope between $S=0.6$ f.u. and $S=0.09$ f.u., as given in Table $I$. The dashed line is the predicted relation, obtained from the $408 \mathrm{MHz}$ counts and the $\alpha(408-5000 \mathrm{MHz})$ distribution. 
made, however, and the scans were spaced at half-beamwidth intervals. The three surveys were:

(a) A 'fast' survey covering 0.97 ster down to a limiting flux density of 0.6 f.u.,* with 136 sources above this limit. Above 0.8 f.u., the earlier survey made in 1968 can be included, giving an area of 1.24 ster and 115 sources above the latter limit.

(b) A 'medium' survey, covering about 0.08 ster down to 0.25 f.u. and containing 38 sources.

(c) A 'deep' survey, covering 0.009 ster in the zones of the Cambridge $5 \mathrm{Cl}$ (Kenderdine et al., 1966) and 5C2 (Pooley and Kenderdine, 1968) surveys and containing 18 sources above $0.09 \mathrm{f} . \mathrm{u}$.

The sources detected in the 'fast' and 'medium' surveys were re-observed and the flux densities were measured with an accuracy of about 0.02 to $0.03 \mathrm{f}$.u., while those of the 'deep' survey sources were obtained from the survey itself with an accuracy of about $0.015 \mathrm{f}$.u. The source counts from the deep survey were corrected for the systematic overestimation of the flux densities caused by random noise and confusion. The correction, which amounted to about $10 \%$ at the limit of the survey, was determined by adding sources of known intensity to the survey map.

The integral number-flux relation derived from the three surveys is shown in Figure 1.

TABLE I

Slope of the $5 \mathrm{GHz} \log N / \log S$ relation

\begin{tabular}{lll}
\hline Flux density range & Slope & Notes \\
\hline$\geqslant 1.0$ f.u. & $-1.76 \pm 0.22$ & 'fast' survey data \\
$\geqslant 0.8$ f.u. & $-1.78 \pm 0.18$ & 'fast' survey data \\
$\geqslant 0.6$ f.u. & $-1.62 \pm 0.15$ & 'fast' survey data \\
0.6 to 0.25 f.u. & $-1.37 \pm 0.21$ & 'fast' survey counts at 0.6 f.u., \\
& & with 'medium' survey counts at \\
0.6 to 0.09 f.u. & $-1.35 \pm 0.14$ & 0.25 f.u. \\
& & 'fast' survey counts at 0.6 f.u., \\
& with 'deep' survey counts at \\
& 0.09 f.u.
\end{tabular}

Table I shows the slope of the $\log N-\log S$ relation for various ranges of the flux density, determined either by the method described by Jauncey (1967), or directly from the independent number counts in two of the surveys.

\section{The Spectral Index Distributions}

The spectral index distribution of the sources in the present survey has been obtained by direct measurements of the flux densities of samples of the sources at 10.6 and $15.3 \mathrm{GHz}$ and also by reference to flux densities at $408 \mathrm{MHz}$ from the Parkes, Bologna, or Cambridge 5C surveys. For comparison of the present survey with the $408 \mathrm{MHz}$

* 1 f.u. $=10^{-26} \mathrm{~W} \mathrm{~m}^{-2} \mathrm{~Hz}^{-1}$. 
surveys, the flux densities at $5 \mathrm{GHz}$ of samples of sources from the above surveys have been measured. In addition, the sources in the $178 \mathrm{MHz}$ North Polar survey of Ryle and Neville (1962), have been observed at $2.7 \mathrm{GHz}$, for comparison with the sources in the $3 \mathrm{C}$ survey.

The results are summarized in Table II. In determining the spectral indices, the

TABLE II

The spectral index distributions

\begin{tabular}{|c|c|c|c|c|c|c|}
\hline $\begin{array}{l}\text { Survey } \\
\text { frequency } \\
\text { (MHz) }\end{array}$ & $\begin{array}{l}\text { Frequency } \\
\text { range } \\
(\mathrm{MHz})\end{array}$ & $\begin{array}{l}\text { Flux density } \\
\text { (f.u.) }\end{array}$ & $\begin{array}{l}\text { Number } \\
\text { of } \\
\text { sources }\end{array}$ & $\begin{array}{l}\text { Median index, } \\
\bar{\alpha}\end{array}$ & $\begin{array}{l}\text { Fraction of } \\
\text { sources with } \\
\alpha>-0.5 \\
\text { (percent) }\end{array}$ & Notes \\
\hline 178 & $178-2695$ & $\begin{array}{l}\geqslant 9 \\
\geqslant 2 \\
\geqslant 0.45 \\
\geqslant 0.25\end{array}$ & $\begin{array}{r}251 \\
410 \\
55 \\
85\end{array}$ & $\begin{array}{l}-0.82 \pm 0.01 \\
-0.84 \pm 0.01 \\
-0.80 \pm 0.02 \\
-0.81 \pm 0.03\end{array}$ & $\begin{array}{l}3.6 \pm 1.2 \\
5.1 \pm 1.1 \\
5.4 \pm 3.1 \\
7.0 \pm 3.5\end{array}$ & $\begin{array}{l}(1) \\
(2) \\
(3) \\
(3)\end{array}$ \\
\hline 408 & $408-5000$ & $\begin{array}{l}\geqslant 6 \\
\geqslant 2 \\
\geqslant 0.5 \\
\geqslant 0.28\end{array}$ & $\begin{array}{r}178 \\
98 \\
96 \\
37\end{array}$ & $\begin{array}{l}-0.77 \pm 0.02 \\
-0.80 \pm 0.02 \\
-0.78 \pm 0.02 \\
-\end{array}$ & $\begin{array}{l}10.5 \pm 2.0 \\
11.2 \pm 3.4 \\
14.6 \pm 3.9 \\
13.5 \pm 6.0\end{array}$ & $\begin{array}{l}(4) \\
(5) \\
(6) \\
(7)\end{array}$ \\
\hline 5000 & $5000-408$ & $\begin{array}{l}\geqslant 1.5 \\
\geqslant 0.7 \\
\geqslant 0.25 \\
\geqslant 0.08\end{array}$ & $\begin{array}{l}25 \\
92 \\
24 \\
15\end{array}$ & $\begin{array}{l}-0.40 \pm 0.10 \\
-0.60 \\
-0.37 \pm 0.09 \\
-0.45 \pm 0.11\end{array}$ & $\begin{array}{ll}60 & \pm 16 \\
54 & \pm 11 \\
58 & \pm 16 \\
60 & \pm 20\end{array}$ & $\begin{array}{l}(8) \\
(8) \\
(8) \\
(8)\end{array}$ \\
\hline 5000 & $5000-10600$ & $\begin{array}{l}\geqslant 0.7 \\
\geqslant 0.08\end{array}$ & $\begin{array}{l}89 \\
18\end{array}$ & $\begin{array}{l}-0.60 \pm 0.05 \\
-0.50 \pm 0.15\end{array}$ & $\begin{array}{l}43 \pm 16 \\
50 \pm 17\end{array}$ & $\begin{array}{l}(8) \\
(8)\end{array}$ \\
\hline 5000 & $5000-15300$ & $\geqslant 0.6$ & 25 & $-0.60 \pm 0.09$ & $50 \pm 15$ & (8) \\
\hline
\end{tabular}

Notes to Table II

All spectral indices were obtained from flux densities at the two frequencies given in column 2; the flux density in column 3 is the lower limit of the sample measured, at the survey frequency. References to data are:

(1) 3CR sources (Kellermann, et al. 1968).

(2) $4 \mathrm{C}$ sources between dec. $0^{\circ}$ and $20^{\circ}$ observed at Parkes (Wills and Bolton, 1969; Shimmins et al., 1966). Complete sample, excluding only confused or extended sources.

(3) Sources in North Polar survey (Ryle and Neville, 1962) (11 cm data is unpublished).

(4) Parkes catalogues (Day et al., 1966; Shimmins et al., 1966); flux densities at $5 \mathrm{GHz}$ mainly measured by us, apart from a few values from Shimmins et al., 1969.

(5) $408 \mathrm{MHz}$ flux densities from Bologna survey (Gruppo ROUB, 1969).

(6) $408 \mathrm{MHz}$ flux densities from Bologna survey (Grueff and Vigotti, 1968).

(7) $408 \mathrm{MHz}$ flux densities from 5C surveys (Kenderdine et al., 1966; Pooley and Kenderdine, 1968). This sample consists of sources which should have been detected in our 'deep' survey, if their spectral indices were $>-0.5$.

(8) Present measurements.

flux density scale used was that of Kellermann et al. (1969). As well as the median index, Table II gives the fraction of sources having spectral indices $\alpha \geqslant-0.5$ in each group, with its statistical uncertainty. The complete distributions are also presented as histograms in Figures 2 to 4.

The slope of the number-flux relation at $5 \mathrm{GHz}$ shows a significant flattening in 
the range 1 to $0.1 \mathrm{f}$.u. The $5 \mathrm{GHz}$ number counts are entirely consistent with the number-flux relation calculated from the Cambridge $408 \mathrm{MHz}$ source counts (Ryle and Pooley, 1968) and from the distribution of the spectral index $\alpha(408-5000 \mathrm{MHz})$ for sources with $S_{408} \geqslant 0.5 \mathrm{f}$.u. (Table II), by the use of the transformation given by Equation 3 of Paper I.

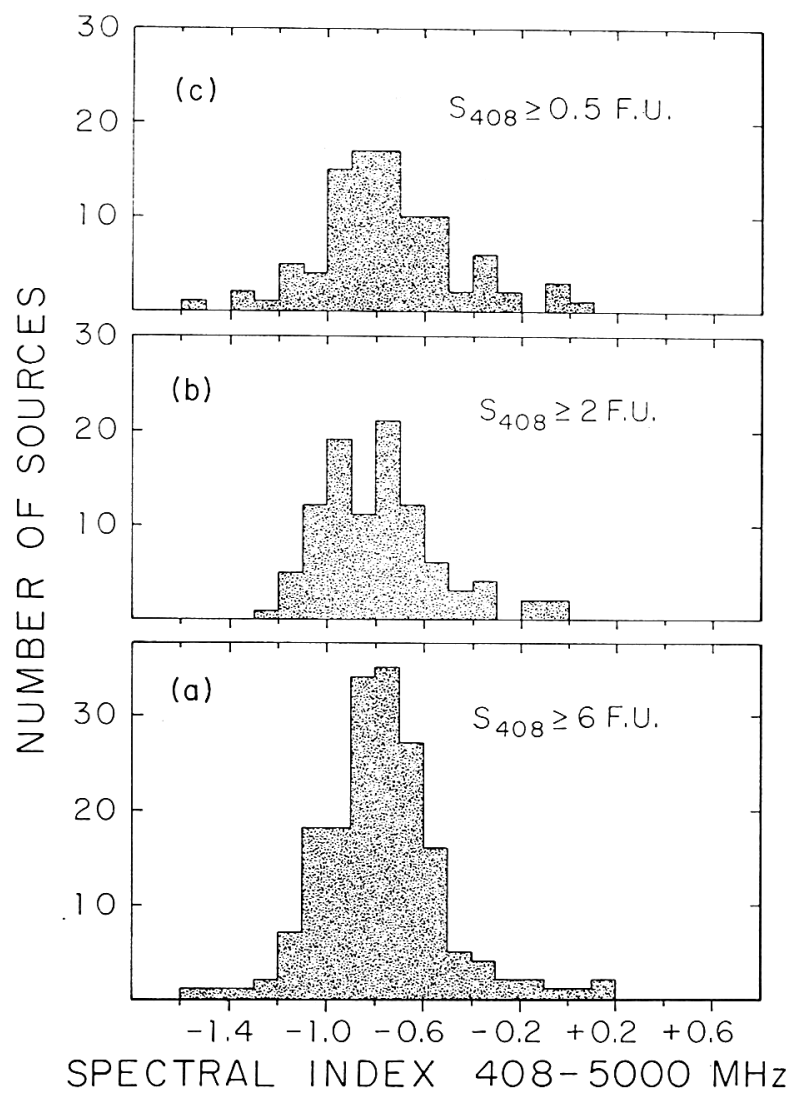

Fig. 2. Distribution of the spectral index $\alpha(408-5000 \mathrm{MHz}$ ) for three $408 \mathrm{MHz}$ surveys: (a) Parkes survey above 6 f.u., (b) Bologna survey above 2 f.u., (c) Bologna survey above 0.5 f.u.

This agreement implies, firstly, that no new population of sources which is not already represented at meter wavelengths appears at centimeter wavelengths; secondly, that the spectral index distribution between $408 \mathrm{MHz}$ and $5 \mathrm{GHz}$ is essentially independent of flux density down to about $0.1 \mathrm{f}$.u. at $408 \mathrm{MHz}$.

The spectral index distributions in Table II show clearly the increase with frequency in the proportion of sources with flat spectra from about $11 \%$ for a survey at $408 \mathrm{MHz}$ to more than $50 \%$ at $5 \mathrm{GHz}$. The source counts at $5 \mathrm{GHz}$ and the spectral distributions $\alpha(5.0-10.6 \mathrm{GHz})$ and $\alpha(5.0-15.3 \mathrm{GHz})$ of Table II may be used to predict the number counts and spectral distribution for surveys at 10.6 and $15.3 \mathrm{GHz}$. Figure 5 shows this 
in the form of two curves, one (solid) giving the source density at a level of $1 \mathrm{f} . \mathrm{u}$. for various surveys and the other (dashed) giving the fraction of sources with $\alpha \geqslant-0.5$, as a function of the survey frequency. Filled circles represent actual surveys; the open circles are predicted values for surveys at 10.6 and $15.3 \mathrm{GHz}$. Clearly, above $10 \mathrm{GHz}$, more than $80 \%$ of sources will have flat spectra and represent the 'normal' source population.

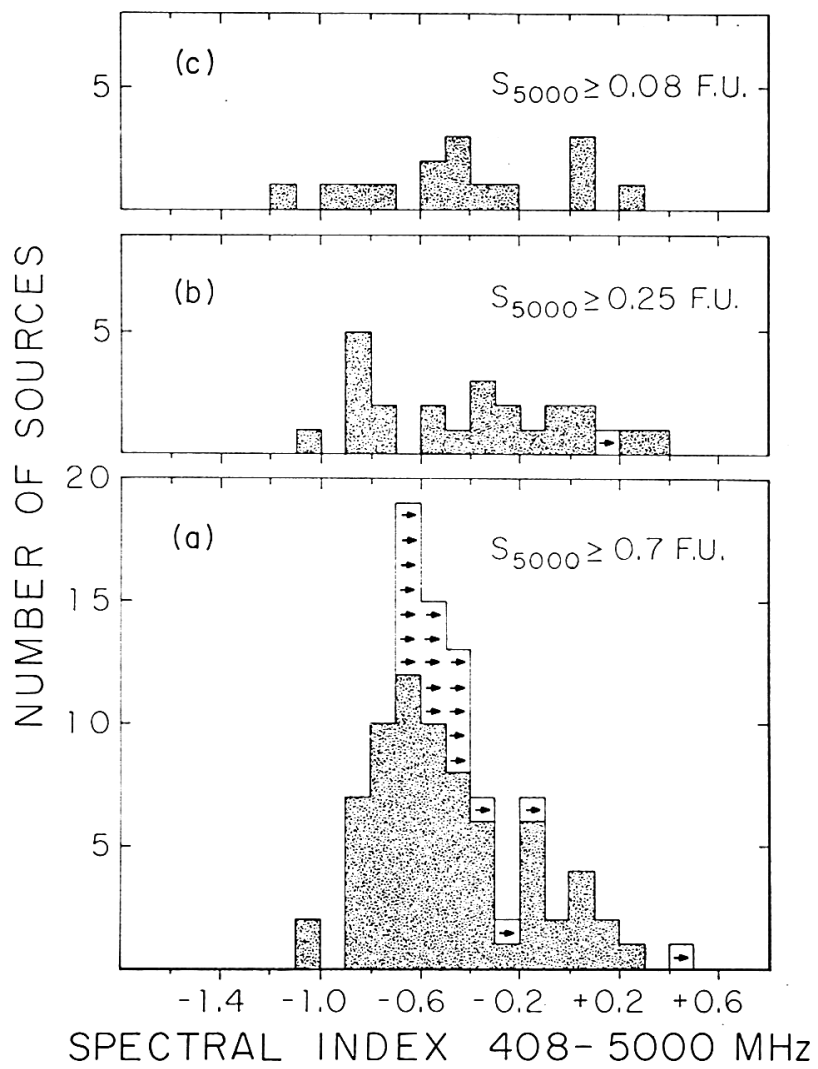

Fig. 3. Distribution of the spectral index $\alpha(408-5000 \mathrm{MHz})$ for three $5 \mathrm{GHz}$ surveys: (a) 'fast' survey above $0.7 \mathrm{f}$.u.; (b) 'medium' survey above 0.25 f.u.; (c) 'deep' survey above 0.08 f.u. Arrows represent lower limits for $\alpha$ for sources not detected by the low-frequency survey. The references to the low frequency data are given in Table II.

The spectral index distribution at $5 \mathrm{GHz}$ is in good agreement with that calculated from the $408 \mathrm{MHz}$ source counts and the distribution $\alpha(408 \mathrm{MHz}-5 \mathrm{GHz})$, using Equation 1 of Paper I: for a sample with $S(5 \mathrm{GHz}) \geqslant 0.8$ f.u., the predicted median index $\alpha(5 \mathrm{GHz}-408 \mathrm{MHz}) \sim-0.38$ and the fraction of sources having $\alpha \geqslant-0.5$ is $59 \%$, both values being close to those observed.

There is no significant change in the spectral distribution at $178 \mathrm{MHz}, 408 \mathrm{MHz}$ or $5 \mathrm{GHz}$ with flux density. There is some suggestion in the 178 and $408 \mathrm{MHz}$ data of an 
increase in the fraction of sources having flat spectra with decreasing flux density, but the change is less than a factor of 2 over a range of flux densities of 20 to 1 . This disagrees with the conclusions of Harris and Kraus (1970), who find that the weaker sources at $408 \mathrm{MHz}$ have considerably steeper spectra than do the strong sources. The number-flux relation for the two classes of source, with $\alpha \sim-0.8$ and

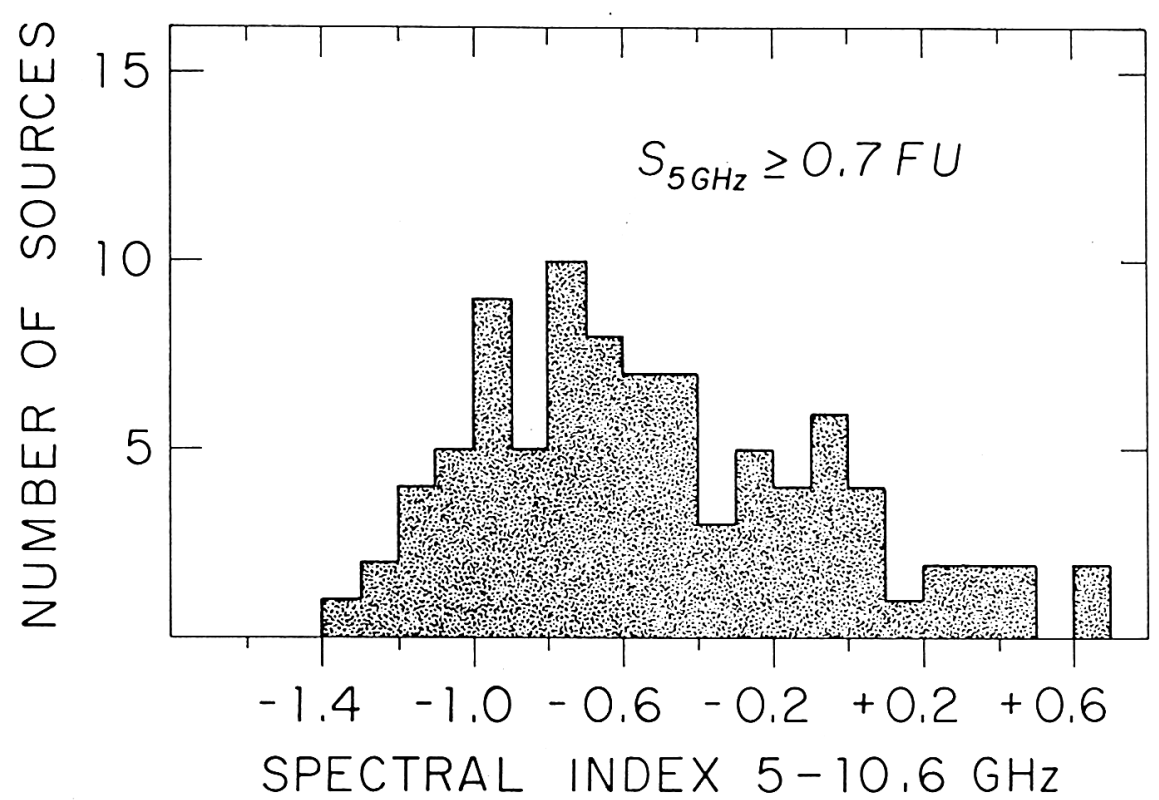

Fig. 4. Distribution of the spectral index $\alpha(5 \mathrm{GHz}-10.6 \mathrm{GHz})$ for sources in the 'fast' survey above $0.7 \mathrm{f} . \mathrm{u}$.

$\alpha \sim 0$ is essentially the same and the difference between the slopes of the respective $\log N-\log S$ curves is less than 0.2 over this range of flux densities.

The lack of any marked change in the spectral index distribution with flux density, or of any dependence of the number counts on the spectral index may imply that the typical redshift at source densities of a few thousand per ster is smaller than the value of 3-4 determined by Longair (1966) at these source densities. Alternatively, the relative luminosity or spatial density of the two types of radio source may vary with redshift in just the right manner to keep the observed source counts the same. This latter explanation requires that sources with flat spectra, which are believed to be young or at an active stage of evolution, become rarer, or less luminous at large redshifts, that is, at earlier epochs.

Further experimental data on both spectral distributions and source counts are clearly required to improve the present statistics, and more detailed theoretical models must be explored. The optimum frequency for the experimental investigation appears to be near $5 \mathrm{GHz}$, where the dispersion in the spectral distribution is largest. 


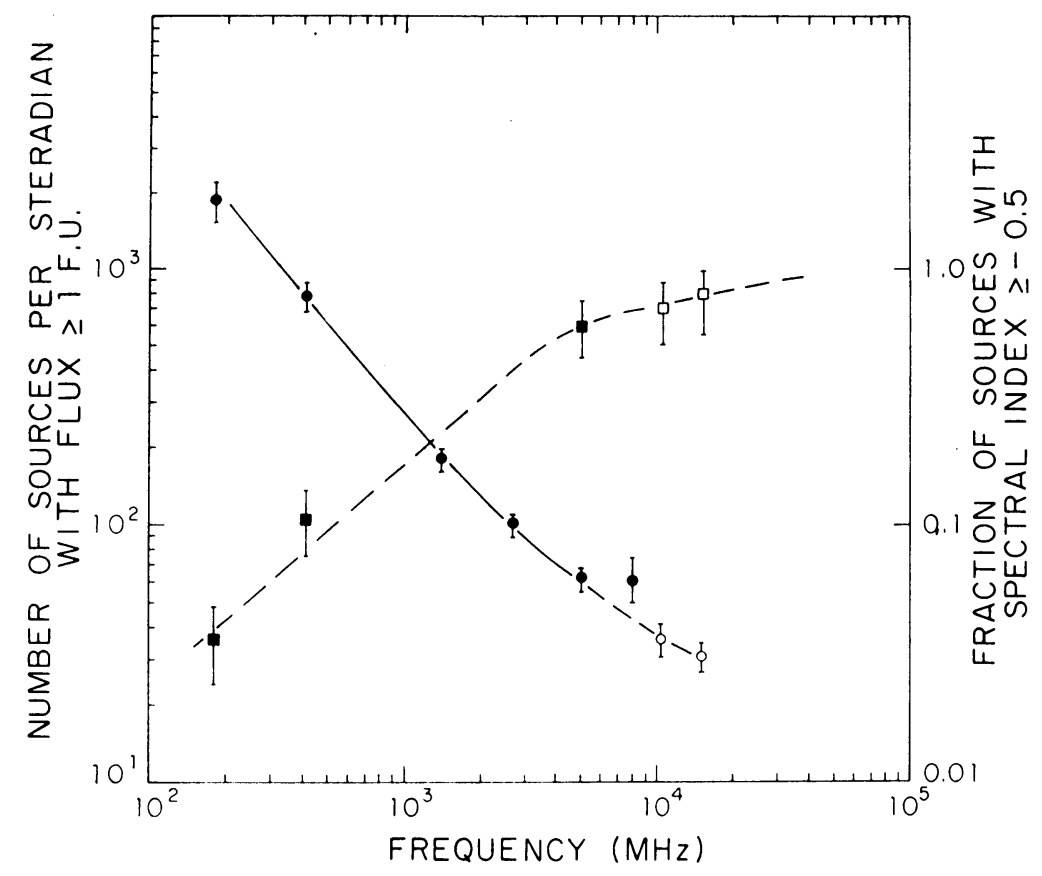

Fig. 5. Number counts and spectral distribution as a function of frequency. The solid data points and solid curve give the source density above a level of $1 \mathrm{f}$.u. for various surveys: $178 \mathrm{MHz}$ (Gower, 1966), $408 \mathrm{MHz}$ (Pooley and Ryle, 1968), $1.4 \mathrm{GHz}$ (Dixon and Kraus, 1968), $2.7 \mathrm{GHz}$ (Shimmins et al., 1968), $5 \mathrm{GHz}$ (present paper), $8 \mathrm{GHz}$ (Brandie, 1970). The unfilled circles represent an extension of the curve based on the $5 \mathrm{GHz}$ counts and the spectral distribution to 10.6 and $15.3 \mathrm{GHz}$. The dashed curve represents the fraction of sources having a spectral index $\alpha \geqslant-0.5$. The spectral index is taken between the survey frequency and $5 \mathrm{GHz}$, except for the $5 \mathrm{GHz}$ survey, where $408 \mathrm{MHz}$ is used as the second frequency. Beyond $5 \mathrm{GHz}$, the curve was calculated from the $5 \mathrm{GHz}$ counts and spectral distribution.

\section{References}

Brandie, G. W.: 1970, Nature 225, 352.

Day, G. A., Shimmins, A. J., Ekers, R. D., and Cole, D. J.: 1966, Australian J. Phys. 17, 340.

Dixon, R. S. and Kraus, J. D.: 1968, Astron. J. 73, 381.

Gower, J. F. R.: 1966, Monthly Notices Roy. Astron. Soc. 133, 151.

Grueff, G. and Vigotti, M.: 1968, Astrophys. Letters 2, 113.

'Gruppo ROUB': 1969, Contribution No. 55 of the Laboratorio Nazionale di Radio-astronomia, University of Bologna.

Harris, B. J. and Kraus, J.: 1970, Nature 227, 785.

Jauncey, D. L.: 1967, Nature 216, 877.

Kellermann, K. I., Pauliny-Toth, I. I. K., and Davis, M. M.: 1968, Astrophys. Letters 2, 105.

Kellermann, K. I., Pauliny-Toth, I. I. K., and Tyler, W. C.: 1968, Astron. J. 73, 298.

Kellermann, K. I., Pauliny-Toth, I. I. K., and Williams, P. J. S.: 1969, Astrophys. J. 157, 1.

Kenderdine, S., Ryle, M., and Pooley, G. G.: 1966, Monthly Notices Roy. Astron. Soc. 134, 189.

Longair, M. S.: 1966, Monthly Notices Roy. Astron. Soc. 133, 421.

Pooley, G. G. and Kenderdine, S.: 1968, Monthly Notices Roy. Astron. Soc. 139, 529.

Pooley, G. G. and Ryle, M.: 1968, Monthly Notices Roy. Astron. Soc. 139, 515.

Ryle, M. and Neville, A. C.: 1961, Monthly Notices Roy. Astron. Soc. 125, 39. 
Shimmins, A. J., Bolton, J. G., and Wall, J.: 1968, Nature 217, 818.

Shimmins, A. J., Day, G. A., Ekers, R. D., and Cole, D. J.: 1966, Australian J. Phys. 19, 649.

Shimmins, A. J., Clarke, M. E., and Ekers, R. D.: 1966, Australian J. Phys. 19, 649.

Shimmins, A. J., Manchester, R. N., and Harris, B. J.: 1969, Australian J. Phys., Suppl. No. 8. Wills, D. and Bolton, J. G.: 1969, Australian J. Phys. 22, 775.

\section{Discussion}

Mrs. Burbidge: What is the typical redshift of the sources?

Pauliny-Toth: In a Euclidean Universe, the typical redshift at source densities of a few thousand per steradian would be less than about $\mathbf{0 . 5}$. 